\title{
Discreteness effects in a reacting system of particles with finite interaction radius.
}

\author{
S. Berti ${ }^{1}$, C. López ${ }^{2}$, D. Vergni ${ }^{3}$ and A. Vulpiani ${ }^{4,5}$ \\ 1 Department of Mathematics and Statistics, \\ University of Helsinki P. O. Box 68, FIN-00014 Helsinki, Finland. \\ 2 Unidad de Física Interdisciplinar, IMEDEA (CSIC-UIB), \\ Campus de la Universitat de les Illes Balears, E-07122 Palma de Mallorca, Spain. \\ 3 Istituto Applicazioni del Calcolo (IAC) - CNR, \\ Viale del Policlinico, 137, I-00161 Roma, Italy. \\ ${ }^{4}$ Dipartimento di Fisica and INFN Sezione Università di Roma "La Sapienza", \\ P.zle Aldo Moro 2, I-00185 Rome, Italy. and \\ 5 INFM-SMC P.zle Aldo Moro 2, I-00185 Rome, Italy.
}

(Dated: April 10, 2007)

An autocatalytic reacting system with particles interacting at a finite distance is studied. We investigate the effects of the discrete-particle character of the model on properties like reaction rate, quenching phenomenon and front propagation, focusing on differences with respect to the continuous case. We introduce a renormalized reaction rate depending both on the interaction radius and the particle density, and we relate it to macroscopic observables (e.g., front speed and front thickness) of the system.

PACS numbers: 05., 05.40.-a, 82.39.-k, 82.40.-g

\section{INTRODUCTION}

Most of the chemical and biological processes that appear in Nature involve the dynamics of particles (e.g., molecules or organisms) that diffuse and interact with each other and/or with external forces [1-3]. If the total number of particles per unit volume, $N$, is very large, a macroscopic description of the system in terms of continuous fields, e.g., density 
or concentration, is usually appropriate. A prototypical model for these reaction-diffusion systems is the Fisher-Kolmogorov-Petrovskii-Piskunov (FKPP) equation [4, 5] describing the spatio-temporal evolution of a concentration

$$
\partial_{t} \theta(x, t)=D \partial_{x x}^{2} \theta+p \theta(1-\theta)
$$

where $D$ is the diffusion coefficient, $p$ is the reaction rate that determines the characteristic reaction time, $\tau=1 / p$, and $\theta(x, t)$ is the concentration field (for simplicity we have assumed one spatial dimension). It is well known $[1,6,7]$ that Eq. (1) admits uniformly translating solutions - fronts- with a speed $v_{0}=2 \sqrt{D p}$ and a front thickness $\lambda_{0}=8 \sqrt{D / p}$. The above results do not depend on the precise details of the reaction rule. Replacing in Eq. (1) $\theta(1-\theta)$ with a convex function $g(\theta)$, with $g(\theta)>0$ for $0<\theta<1, g(0)=g(1)=0$, and $g^{\prime}(0)=1$, $g^{\prime}(1)<0$, one has the same behaviour for $v_{0}$ and $\lambda_{0}[8]$.

On the other hand, if the number of particles per unit volume is not very large, the continuous description could not be appropriate. In such a case, one can consider a discrete particle model with $N$ particles whose positions $\mathbf{x}_{\alpha}(t)$ evolve according to the Brownian motion

$$
\frac{d \mathbf{x}_{\alpha}(t)}{d t}=\sqrt{2 D} \boldsymbol{\eta}_{\alpha}, \alpha=1, \ldots, N
$$

where $\boldsymbol{\eta}$ is a white noise term. Moreover each particle is characterized by a color $C_{\alpha}(t)$ which determines the particle type. The model is completed by the reaction rule between particles. In order to obtain an autocatalytic reaction

$$
A+B \stackrel{p}{\longrightarrow} 2 B,
$$

one can consider just two types of particles $C=0$ (unstable) and $C=1$ (stable), that correspond to the species $A$ and $B$, respectively, with the following dynamics: particles of type 1 always remain 1 , and particle 0 changes to 1 with a given probability that depends both on $p$, the reaction rate, and on how many 1 particles are around it. It is not difficult to realize 
that in a suitable continuum limit, Eq. (1) gives the evolution of the color concentration of this microscopic system (see Section II). The aim of this work is precisely to study the case in which the density of individuals is small, and therefore the discrete nature of the system can play a role $[9,10]$.

Several approaches have been adopted to investigate the relevance of the correction to the continuum limit. On one side, it has been assumed that the dynamics of the system is given by deterministic macroscopic equations like Eq. (1), and a noise term, of order $1 / \sqrt{N}$, which takes into account the microscopic fluctuations originated by the finite number of particles [11]. On the other side, following the work of Brunet and Derrida [12], this problem has been successfully studied by using a cutoff at the density value $1 / N$ for the continuous field equations. This has been employed to determine corrections to some front properties in FKPP-like equations (see [13] for a review). In particular, it has been shown that the deviation from the continuum value of the front speed is of the order $1 /(\ln N)^{2}$, which is rather significant [12].

More recently, Kaneko and coworkers [14] analyzed the dynamics of some chemical reactions, studying the influence of the molecular discreteness. They identify typical length scales in the system which may separate the continuum behavior from the discretenessinfluenced one. They report transitions to a novel state with symmetry breaking that is induced by discreteness, but they do not investigate the features of front propagation properties in terms of the number of particles. A crucial quantity is the so-called Kuramoto length, $l_{K}=\sqrt{2 D \tau}$, which is proportional to the front thickness and measures the typical distance over which an unstable particle diffuses during its lifetime (note that $\tau=1 / p$ can be interpreted as the average lifetime, i.e., the time particles live before they react). In some situations, especially when there is a propagating front, if the typical distance among particles is much smaller than $l_{K}$, the concentration of the particles can be regarded as continuous. On the other hand, when there are not many particles within a region of size 
$l_{K}$, discreteness effects should be taken into account [14].

In our work we study the interplay between length scales in the problem, our principal aim being to explain the effects of the discrete nature of the system on properties like reaction rate, quenching and front speed. Differently from most of the works in discrete reaction-diffusion systems, we do not consider a lattice model: particles diffusively move in space and interact when their distance is smaller than an interaction radius $R$, which corresponds to a natural length-scale appearing in many chemical and biological systems [10, 15]. We study several properties of the system as a function of $R$, realising, via comparison of different length-scales, when the effects of discreteness have a dominant role. As expected, the continuum limit is described by the FKPP equation. Nevertheless we remark that in order to have the proper continuum limit it is not sufficient to have a very large density of particles. We discuss the problem in the framework of chemical reaction dynamics, but everything can be thought in the context of population dynamics.

The Paper is organized as follows. In the next section we present the particle model for the autocatalytic reaction. In section III we study the renormalized reaction rate of the system when particles of both types are in a closed vessel, initially uniformly random distributed in space. In section IV we study quenching phenomena when $B$ particles can turn into $A$ particles; this causes the emergence of new properties of the model that will be studied in detail. Then, in Sect. $\mathrm{V}$ we investigate the front properties of the model (by choosing a proper initial distribution and considering an infinite system in the propagation direction), mainly in terms of the interaction radius of the system. Section VI presents our conclusions. 


\section{MODEL}

Consider $N$ particles in a two-dimensional box of size $L_{x} \times L_{y}$. Each particle is identified by its position, $\mathbf{x}_{\alpha}(t)$, and its color, $C_{\alpha}(t)$, indicating the particle type. To specify the dynamics it is necessary to give the evolution rule for the position and the interaction rule between particles (chemistry). Space will be considered continuous while time will be discrete (with time step $\Delta t$ ). However its value, if small enough, it is not relevant. Particle dynamics is synchronous, i.e., all particle properties are updated at the same time.

The position evolution is given by

$$
\mathbf{x}_{\alpha}(t+\Delta t)=\mathbf{x}_{\alpha}(t)+\sqrt{2 D \Delta t} \mathbf{u}_{\alpha}(t), \quad \alpha=1, \ldots, N
$$

where $D$ is the diffusion coefficient, $\mathbf{u}_{\alpha}(t)=\left(u_{\alpha, 1}(t), u_{\alpha, 2}(t)\right)$ are stochastic Gaussian variables with the properties $\left\langle\mathbf{u}_{\alpha}(t)\right\rangle=0$ and $\left\langle u_{\alpha, i}(n \Delta t) u_{\alpha, j}(m \Delta t)\right\rangle=\delta_{i j} \delta_{\alpha \beta} \delta_{m n}$, i.e., particles perform a discrete-time Brownian motion.

As already mentioned, to model an autocatalytic reaction (3), we consider two kinds of particles: type $A$ particles, $C_{\alpha}=0$ (unstable), and type $B$ particles, $C_{\alpha}=1$ (stable). The chemical evolution of the particles is given by the following stochastic process:

- if $C_{\alpha}(t)=0$ then $C_{\alpha}(t+\Delta t)=1$ with probability $P_{A B}=W_{A B} \Delta t$;

- if $C_{\alpha}(t)=1$ then $C_{\alpha}(t+\Delta t)=1$.

The probability (per unit time) $W_{A B}$ depends on the number of stable particles within the interaction radius. In fact, in the continuum limit, the autocatalytic reaction (3) is expected to obey the mass action law $\frac{\mathrm{d} \Theta_{A}}{\mathrm{~d} t}=-p \Theta_{A} \Theta_{B}$, where $\Theta_{A}$ and $\Theta_{B}$ are the concentrations of particles $A$ and $B$, respectively, with $\Theta_{A}+\Theta_{B}=1$. The probability that a particle $A$ changes into a $B$ particle is assumed to be

$$
W_{A B}=p \frac{N_{R}(B)}{\left\langle N_{l o c}(R)\right\rangle}=p \frac{N_{R}(B)}{\pi R^{2} \rho} .
$$


where $N_{R}(B)$ indicates the number of $B$ particles within the interaction radius $R$ around the given particle $A,\left\langle N_{l o c}(R)\right\rangle$ is the spatial average number of particles (of any type) in a radius $R$, and $\rho=N /\left(L_{x} L_{y}\right)$ is the density of particles.

We discuss in the following that in a suitable limit the previous probabilistic rule converges to the FKPP equation. Let $N(A, t)$ and $N(B, t)$ be the total number of $A$ and $B$ particles, respectively; of course $N=N(A, t)+N(B, t)$ is constant. The dynamics of the number of $\mathrm{B}$ particles is given by the discrete stochastic process

$$
N(B, t+\Delta t)=N(B, t)+\sum_{k \in N(A, t)} y_{k}
$$

where $k$ is the index identifying $A$ particles and $y_{k}$ is a discrete random variable which is 1 with probability $\Delta t W_{A B}$ (when the particle $A$ changes into a $B$ particle), and is 0 with probability $1-\Delta t W_{A B}$ (when the particle $A$ remains $A$ ). For the expected value of $N(B, t)$, one has

$$
\begin{gathered}
E(N(B, t+\Delta t))=E(N(B, t))+E(N(A, t)) p \frac{E\left(N_{R}(B, t)\right)}{\pi R^{2} \rho} \Delta t= \\
=E(N(B, t))+p[N-E(N(B, t))] \frac{E\left(N_{R}(B, t)\right)}{\pi R^{2} \rho} \Delta t
\end{gathered}
$$

After a little algebra we obtain

$$
\frac{\mathrm{d}}{\mathrm{d} t} \Theta_{B}(t)=\lim _{\Delta t \rightarrow 0} \frac{\Theta_{B}(t+\Delta t)-\Theta_{B}(t)}{\Delta t}=p\left(1-\Theta_{B}(t)\right) \frac{E\left(N_{R}(B, t)\right)}{\pi R^{2} \rho} .
$$

where $\Theta_{B}=E(N(B, t)) / N$ indicates the expected average concentration of B particles. In the case of an infinite number of spatially premixed particles the last term on the righthand-side of the above relation becomes $\Theta_{B}(t)$ and we finally obtain the FKPP equation for the homogeneous case:

$$
\frac{\mathrm{d}}{\mathrm{d} t} \Theta_{B}(t)=p\left(1-\Theta_{B}(t)\right) \Theta_{B}(t)
$$

In general, under non-premixed spatial conditions and/or a small density, $\frac{E\left(N_{R}(B, t)\right)}{\pi R^{2} \rho} \neq \Theta_{B}$ and the system cannot be described by the FKPP dynamics. 
Concerning the relevant length scales of the system one can identify the following ones:

i) the mean nearest neighbour distance between particles, $d_{m}=\frac{1}{2 \sqrt{\rho}}=\sqrt{\frac{L_{x} L_{y}}{4 N}}$, ii) the interaction radius of the model, $R$, iii) the Kuramoto length scale, $l_{K}$ and iv) the size of the system $L$. It is expected that the continuum limit is obtained when $d_{m} \ll R \ll l_{K} \ll L$. While the scale separation between $d_{m}$ and $L$ can be easily achieved, in many situations it might happen that the condition $R \ll l_{K}$ is not verified, or that $R$ is of the same order of $d_{m}$. In this case the evolution of the system could be very different from that of the continuous FKPP limit. It is the objective of this work to investigate some properties of the model in this regime.

Before starting with the discussion of the numerical results, some comments follow about the role of diffusion. Since we introduce the natural length-scale of the interaction, $R$, a diffusive time related to this distance arises $t_{D}(R)=R^{2} / D$. When this time is much smaller than the reaction time $\tau=1 / p$ the system is locally homogeneized before reaction happens. In order to focus on the reaction properties rather than on the diffusive effects we work in the limit $t_{D} \gg 1 / p$.

\section{PREMIXED PARTICLES IN CLOSED BASINS}

Firstly we study the model in a closed vessel, where, as initial condition, particles of both types are premixed and uniformly randomly distributed in space. In such a case, the system evolution necessarily ends with the complete filling of the box with type $B$ particles. Therefore the most significant physical quantity is the filling rate of particles $B$, which is related to the reaction rate. We proceed by fixing the value of $R$ and varying $N$ in order to explore different situations: a) continuum limit, $d_{m} \ll R$; b) the effect of the discreteness, $d_{m} \gtrsim R$. In this case, at variance with front propagation properties discussed in Sect. V, we will see that the Kuramoto length does not play a fundamental role. The basic reason for 
this is the spatially random distribution of particles.

We adopt periodic boundary conditions on a square domain of side $L_{x}=L_{y}=1$; the reaction rate is set to $p=1$; and averages are numerically computed over a large number of noise realizations.

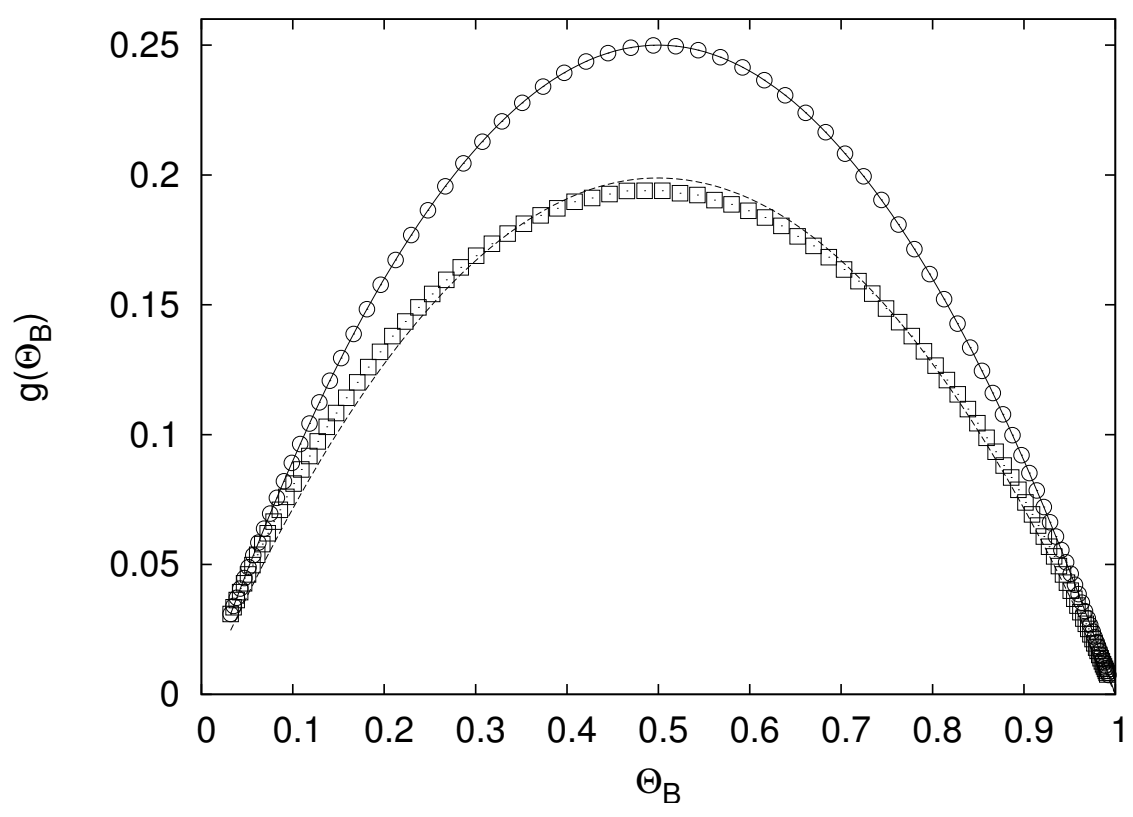

FIG. 1: The growth rate $g\left(\Theta_{B}\right)$ vs $\Theta_{B}$ (see Eq. 9) for $N=1000$ ( $\square$ ), and $N=100000$ (०) with $p=1, D=0.001, R=0.05$; the initial concentration corresponds to $97 \%$ of type $A$ particles and $3 \%$ of $B$ particles, uniformly distributed. The solid and the dashed lines correspond to the quantity $p_{R}(N) \Theta_{B}\left(1-\Theta_{B}\right)$, where $p_{R}(N)$ is fitted from numerical results of the discrete particle model: solid-line is for $p_{R}(N)=1$ and dashed-line for $p_{R}(N)=0.8$.

Since particles are well premixed, the process is spatially homogenous, and we may assume that the growth rate of $\Theta_{B}(t)$, (see Eq. 7) is

$$
g\left(\Theta_{B}\right)=p\left(1-\Theta_{B}(t)\right) \frac{E\left(N_{R}(B, t)\right)}{\pi R^{2} \rho}
$$

In the case of large particle density one expects that $g\left(\Theta_{B}\right)=p \Theta_{B}\left(1-\Theta_{B}\right)$, therefore it is natural to assume that for finite $N$ one can replace eq. (9) with

$$
g\left(\Theta_{B}\right)=p_{R}(N) \Theta_{B}\left(1-\Theta_{B}\right)
$$


where $p_{R}(N)$ is a renormalized reaction rate of the discrete particle model. In this way the evolution of $\Theta_{B}$ is given by an FKPP equation with a renormalized ( $R$ - and $N$ - dependent) reaction probability, where $\tau_{R}(N)=1 / p_{R}(N)$ is the renormalized reaction time for the system. Note that $p_{R}(N)$ contains all of the dependence of our system on the interaction radius and the number of particles and, therefore, it is the proper quantity to study the influence of the discreteness in the model.

In Figure 1 it is shown, for a given $R$, the function $g\left(\Theta_{B}\right)$, with the approximation of Eq. (10) for two different values of $N$. With the appropriate $p_{R}(N)$ value, the fit is rather good and, for large $N, p_{R}(N) \rightarrow p$.

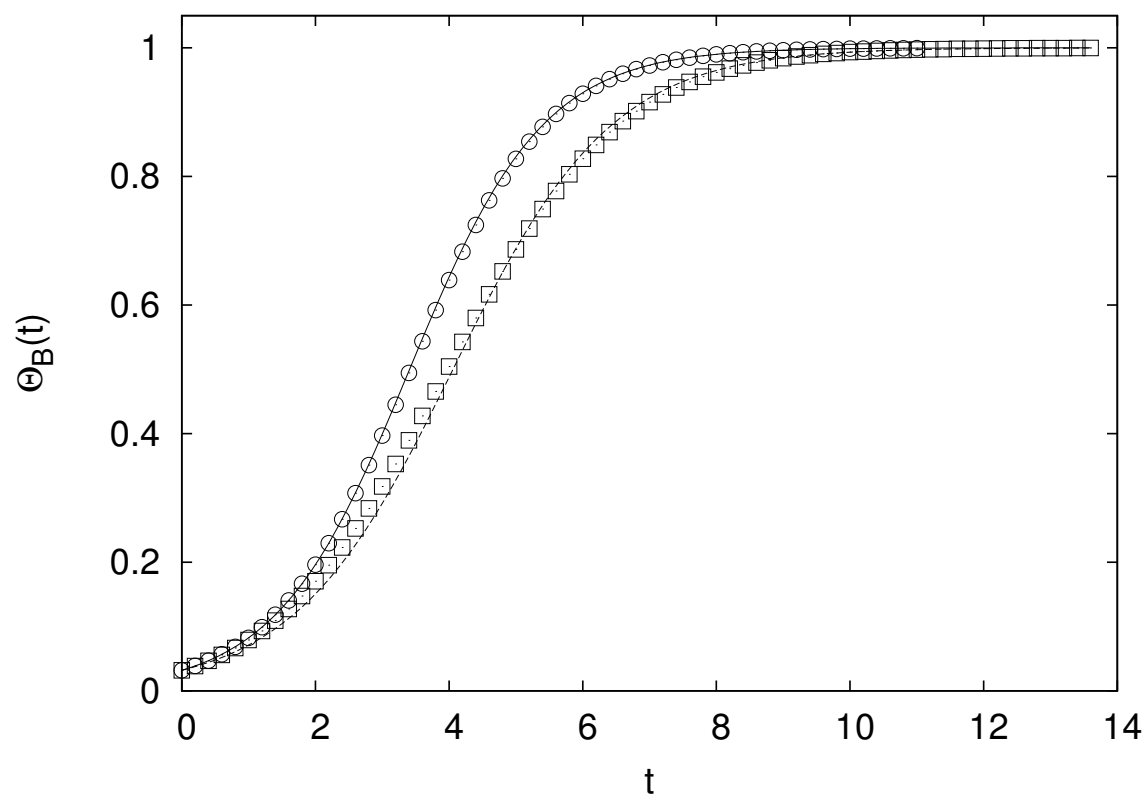

FIG. 2: $\Theta_{B}(t)$ versus $t$ in the same experimental asset (and using the same symbol) of Fig. 1. The solid and the dashed lines correspond to the fit of Eq. (11) on the experimental measures. In particular solid-line is for $p_{R}(N)=1.0$ and dashed-line for $p_{R}(N)=0.82$.

The equation $\frac{\mathrm{d} \Theta_{B}}{\mathrm{~d} t}=p_{R}(N) \Theta_{B}\left(1-\Theta_{B}\right)$ can be easily solved:

$$
\Theta_{B}(t)=\frac{\Theta_{B}(0) e^{p_{R}(N) t}}{1+\Theta_{B}(0)\left(e^{p_{R}(N) t}-1\right)}
$$

Thus looking at the evolution of $\Theta_{B}=E(N(B, t)) / N$ and using Eq. (11) we have a value 
of $p_{R}(N)$ which is, in principle, different from the one in Eq.(10). However, the two values are rather close, and in the following we will present results only for the last one, obtained from Eq.(11). As an example, in Fig. 2, we show $\Theta_{B}(t)$ versus time obtained from the numerical simulation of the particle model, and the best fit using Eq. (11) from which a value of $p_{R}(N)$ comes out. As previously shown in Figure 1, for large $N$ the value of $p_{R}(N)$ goes to the continuum limit $p$. In Figure 3, where the renormalized reaction probability versus $N$ is plotted, one sees that the continuum limit, $p_{R}(N)=p$ is, as expected, obtained with good accuracy for large $N$ values. This corresponds to $d_{m} \ll R$, while for values of $N$ such that $d_{m}$ is comparable or smaller than $R$ the continuous description becomes inaccurate.

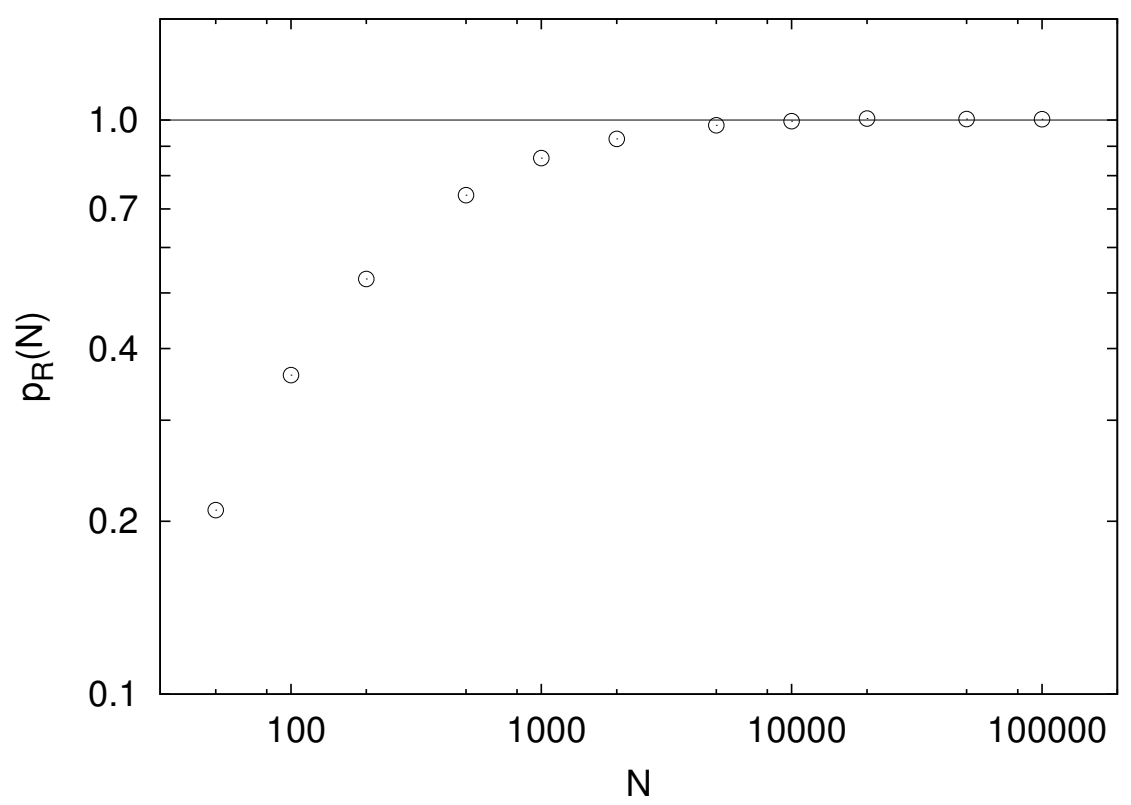

FIG. 3: $p_{R}(N)$ versus $N$. The parameters are the same as in Fig. 1. In particular, $R=0.05$, and the continuum limit is obtained for $N \approx 1000$ for which $d_{m} \approx 0.015$.

More important for our purpose is the behavior of $p_{R}(N)$ versus $R$. With a fixed total number of particles, $N$, and a well premixed initial condition, we compute $p_{R}(N)$ varying the interaction radius $R$ (see fig. 4). We observe that in the continuum limit $\left(d_{m} \ll R\right)$ we recover $p_{R}(N)=p$. For small $R$, such that $d_{m}>R, p_{R}(N)$ seems to reach a constant value, which is around $30 \%$ smaller than the FKPP one. 


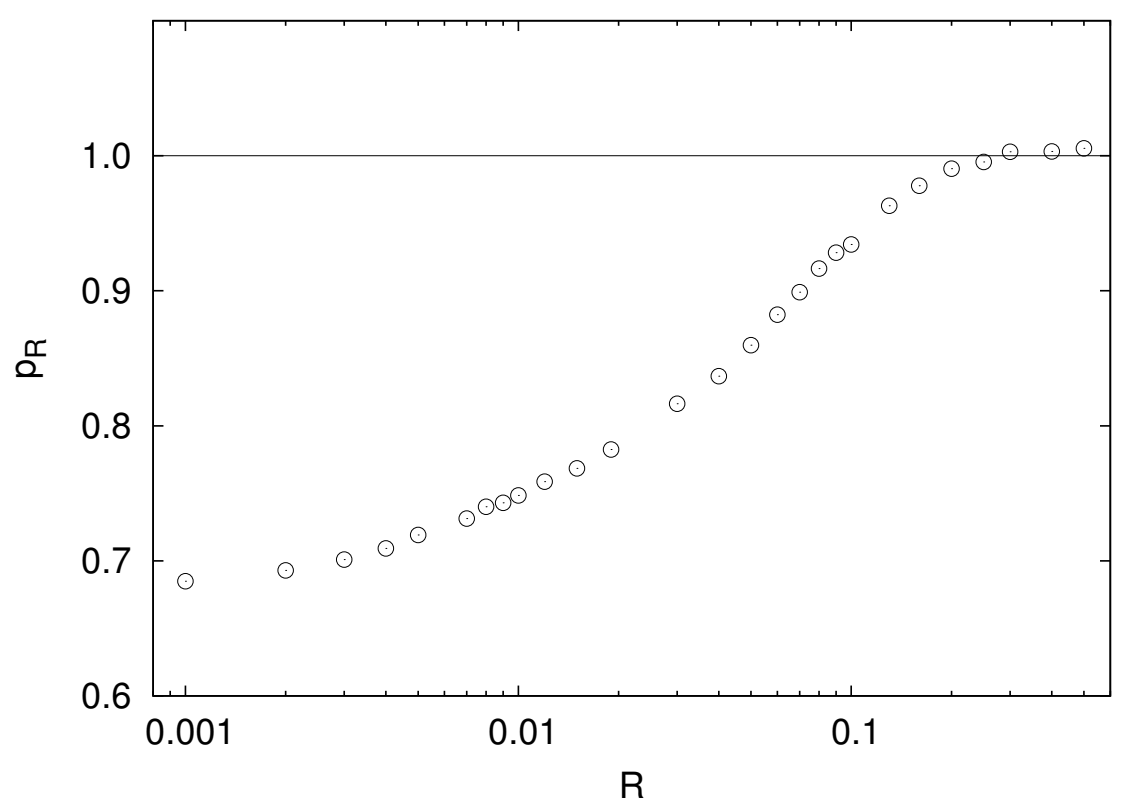

FIG. 4: The renormalized reaction probability $p_{R}(N)$ versus $R$, using the fit of equation (11). $N=1000, l_{K}=0.045$ and $d_{m}=0.0158$.

Few words have to be spent about the difference between the large $N$ limit (of Figure 3 ) and the large $R$ limit of Figure 4 . For the problem under discussion one has the same behavior of the continuum limit if $d_{m} \ll R$ irrespective of the value of the Kuramoto length. For example, in Fig. 4 one has $l_{K} \approx 0.045$ which is much smaller than the values of $R$ for which the continuum limit holds. On the other hand, in the study of front properties we will see that the scenario is different and $l_{K}$ can play a relevant role.

Now we want to discuss the dependence of the previous results on the chosen initial condition. In Figure 5 we compare $p_{R}(N)$ in the premixed case, and when particles are initially separated in space. Indeed, if the premixed particle condition is relaxed, and the system is initially prepared with different spatial distributions of particles, the renormalized reaction probability significantly changes, since $E\left(N_{R}(B, t)\right)$ strongly depends on the particle configuration (see the inset in Figure 5). 


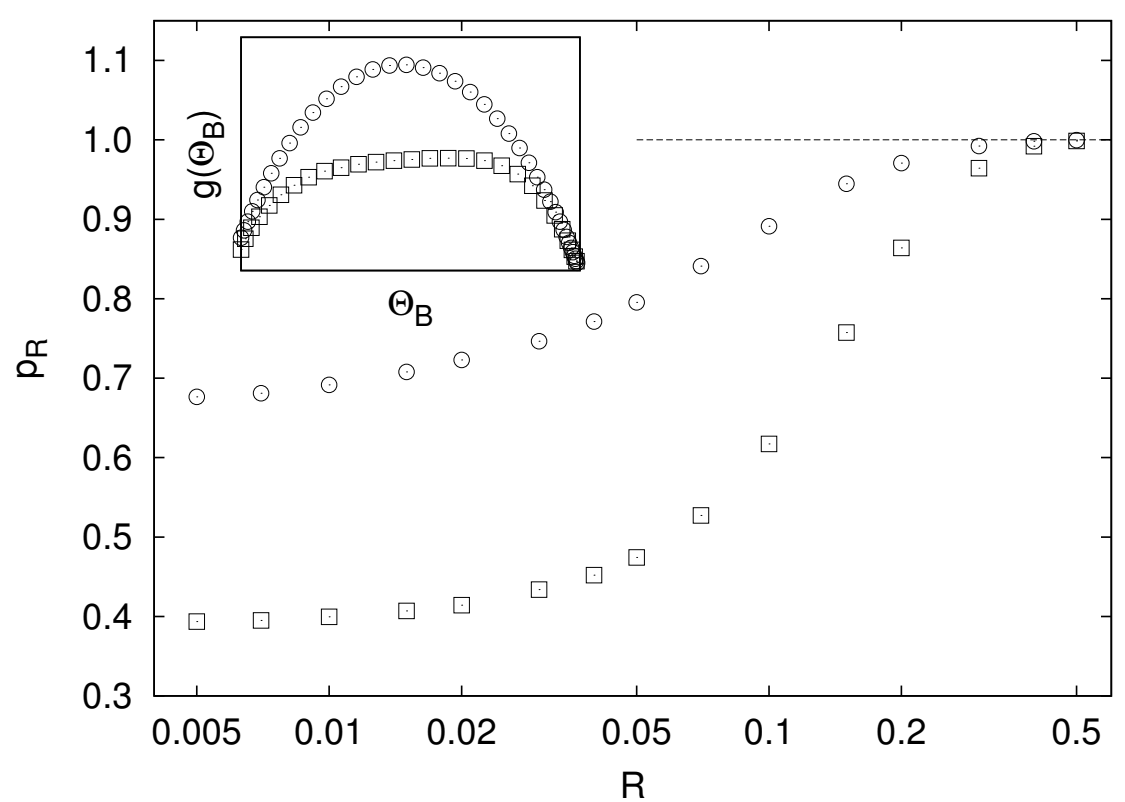

FIG. 5: $p_{R}(N)$ versus $R$ for different initial conditions: (o) premixed, ( $\square$ ) B particles on the left of the system and A particles on the right. The parameters are the same as in fig. 4. In the inset we plot the corresponding $g\left(\Theta_{B}\right)$ vs $\Theta_{B}$ for $R=0.05$; (०) premixed, and ( $\square$ ) initially separated particles.

\section{POSSIBILITIES OF QUENCHING.}

Studies on the quenching phenomenon [16] shows that in a continuous reaction-diffusion system in presence of an advectin velocity field, and with a reaction term of ignition type, i.e. $g(\Theta)=0$ for $\Theta<\Theta_{c}$, for a suitable size of the "hot" region, there is no propagating front, and the reaction quenches. On the contrary, in a continuous FKPP system (1) quenching phenomena do not appear [17]. Here we show that in a particle description of an FKPP system quenching can occur.

Still considering premixed particles in a closed vessel, let us introduce the possibility that a stable particle $(B)$ can turn into an unstable one $(A)$. That is, beyond the autocatalytic 
reaction (3), we introduce a new reaction

$$
B \stackrel{q}{\longrightarrow} A
$$

where $q$ is its rate. Therefore we have the following reaction rules:

- if $C_{\alpha}(t)=0$ then $C_{\alpha}(t+\Delta t)=1$ with probability $P_{A B}=W_{A B} \Delta t$

- if $C_{\alpha}(t)=1$ then $C_{\alpha}(t+\Delta t)=0$ with probability $Q_{B A}=W_{B A} \Delta t$

$W_{A B}$ is the same of the previous section, while $W_{B A}=q$ does not depend on the interaction radius $R$, since it is a single particle property.

The renormalized description of this model is given by

$$
\frac{d \Theta_{B}(t)}{d t}=p_{R}(N) \Theta_{B}\left(1-\Theta_{B}\right)-q \Theta_{B}
$$

whose solution is

$$
\Theta_{B}(t)=\Theta_{A S} \frac{\Theta_{B}(0) e^{\Lambda t}}{\Theta_{A S}+\Theta_{B}(0)\left(e^{\Lambda t}-1\right)} .
$$

with $\Lambda=p_{R}(N)-q$ and $\Theta_{A S}=1-q / p_{R}(N)$. Two different scenarios now appear. If $p_{R}(N)<q$ for all $N$ the reaction finishes. On the other side, when $p_{R}(N)>q$ we have a similar behavior as in the case with $q=0$. In fig. 6 we show $\Theta_{B}$ vs $t$ for different values of $R$. It is apparent that for large $R$ the system behaves similarly to the case $q=0$ (including the continuum limit for the long time value of the concentration, $1-p / q$, for large $R$ ). However, for $R$ small enough the concentration asymptotically vanishes, that is we have a quenching phenomenon. In Fig. 7 where we plot $\Lambda$ vs $R$ (obtained analogously to Fig. 4, i.e., fitting the analytical solution to the numerical results). For large $R$ we approach the continuum limit and $\Lambda \rightarrow p-q$, while for small $R$ we have quenching corresponding to negative values of $\Lambda$.

This is a relevant result, entirely due to the role of the interaction radius, $R$, which reflects the discrete character of the model in the quenching mechanism. Let us note that, 


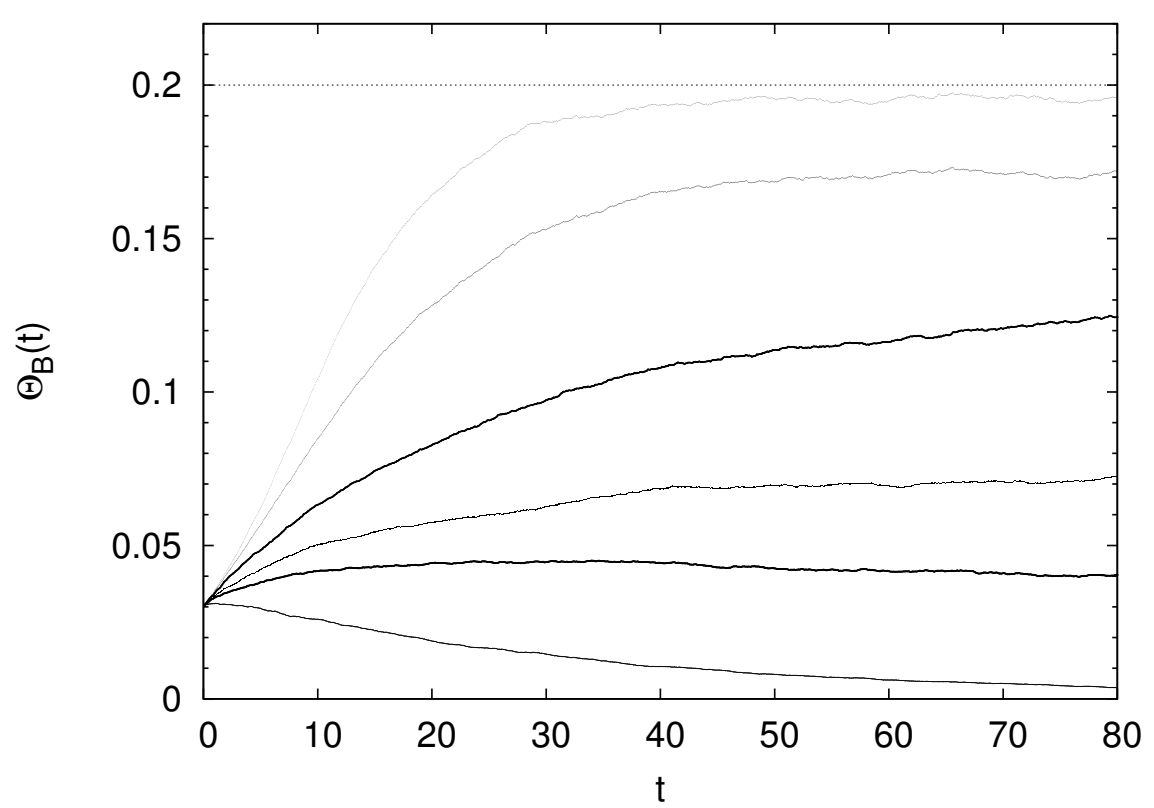

FIG. 6: Time evolution of $\Theta_{B}(t)$ for $R=0.5,0.1,0.05,0.03,0.02,0.005$ (from top to bottom), $N=1000, D=0.001$; the straight line is the continuum limit asymptotic value $1-q / p$, with $p=1, q=0.8$. For $R<0.02$ the reaction quenches.

at variance with the results in the previous section (which are just quantitative changes with respect to the continuous equation), now the discrete nature of the system is able to produce a feature (the quenching) which is absent in the continuum limit [17].

\section{FRONT PROPERTIES}

In the previous sections we have studied the dynamics of interacting particle systems in a closed container. We now focus on a different configuration, corresponding to well-separated chemicals in an open domain, and we investigate evolution properties, such as front speed and thickness $[18,19]$, in terms of the interaction radius.

In this section we take $L_{y}=1, L_{x}=5$, with periodic boundary conditions in the $y$ direction, and rigid walls in the $x$ direction. The burnt (type $B$ ) particles are initially concentrated in the leftmost part of the system, so that a propagating reaction front, from 


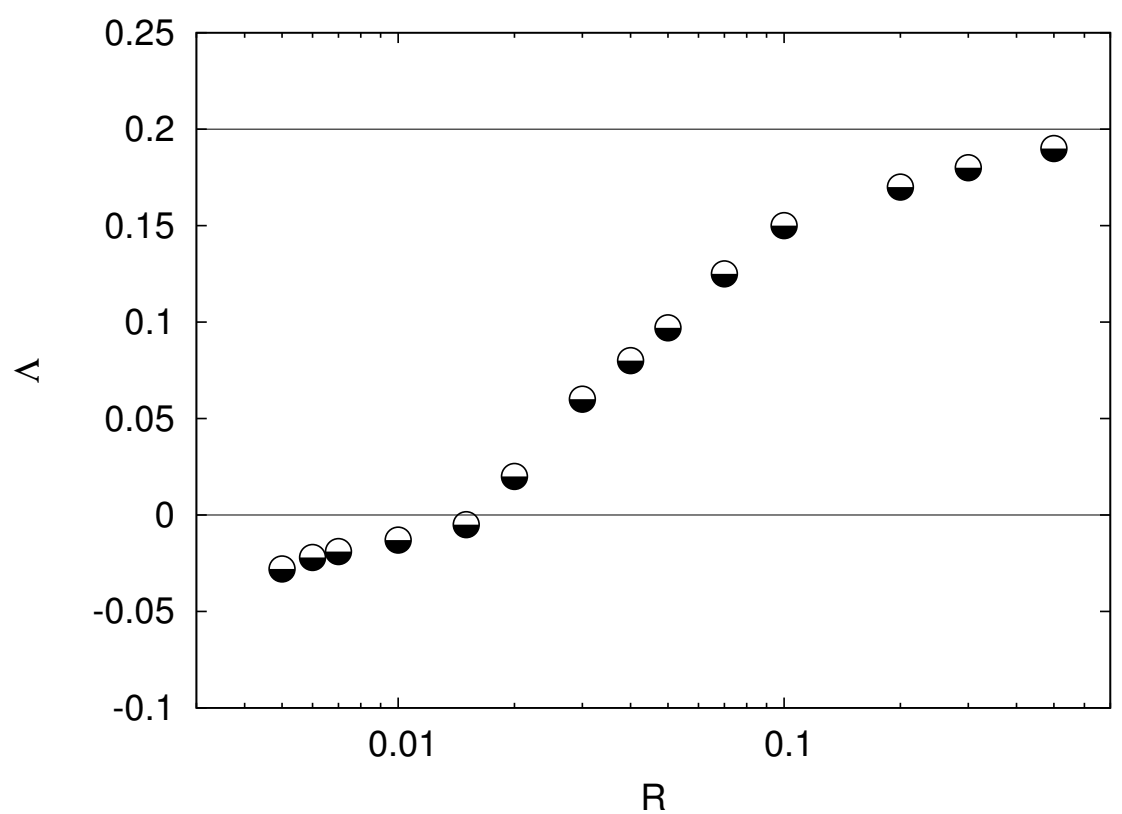

FIG. 7: Inverse characteristic time of the reacting process $\Lambda=p_{R}(N)-q$ as a function of the interaction radius $R$ (with $p=1, q=0.8$ ); $N=1000, D=0.001$. For large values of $R, \Lambda$ tends to the continuum limit value $p-q$; for $R<0.02 \Lambda$ becomes negative, highlighting the emergence of the quenching phenomenon.

left to right, develops. The reaction term we use is just the autocatalytic one (3), i.e., $q=0$. We separately study the front speed and the front thickness.

\section{A. Front speed}

We can define the instant front position as

$$
x_{\mathrm{f}}(t)=L_{x} \frac{N_{B}(t)}{N}
$$

and the front speed:

$$
v_{\mathrm{f}} \simeq \frac{x_{\mathrm{f}}(t)}{t}
$$

which is computed after a transient and before the complete saturation is approached. In Fig. 8 we show $x_{\mathrm{f}}(t)$ vs time for different values of $R$. The $v_{\mathrm{f}}$ is obtained as the slope of the best fit to the curves in the proper interval. 


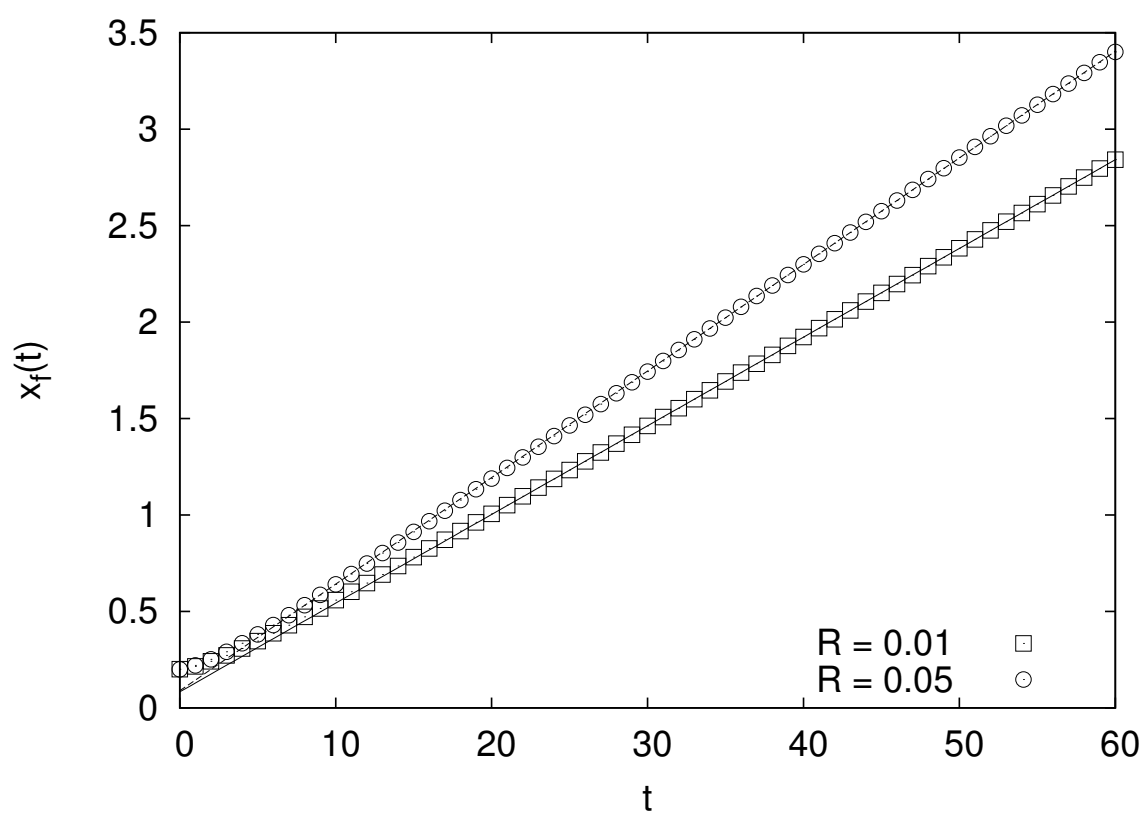

FIG. 8: $x_{\mathrm{f}}$ vs $t$. The system parameters are: $L_{x}=5, L_{y}=1, D=0.001, N=5000$, and the initial number of $B$ particles is 200 . The slopes of the straight lines represent the front speed.

We expect that, via the renormalized description of the FKPP equation, that is Eq. (1) with $p$ replaced by $p_{R}(N)$, the front speed of the particle model at varying $R$ should be

$$
v_{0}=2 \sqrt{D p_{R}(N)}
$$

We saw that in closed basins different initial conditions on the distribution of particles select different $p_{R}(N)^{\prime}$ 's, see Figure 5. Therefore, for the study of front propagation the proper $p_{R}(N)$ is that one computed in the case of initially separated particles distribution (symbol ( $\square$ ) in Figure 5). The numerical results, reported in Fig. 9, confirm our prediction at least for small $R$, i.e., the front speed behaves as in the FKPP case (Eq. (17)).

However, the large discrepancy observed for large $R$ cannot be explained by a simple difference in the initial particle distribution. This difference arises because the interaction 


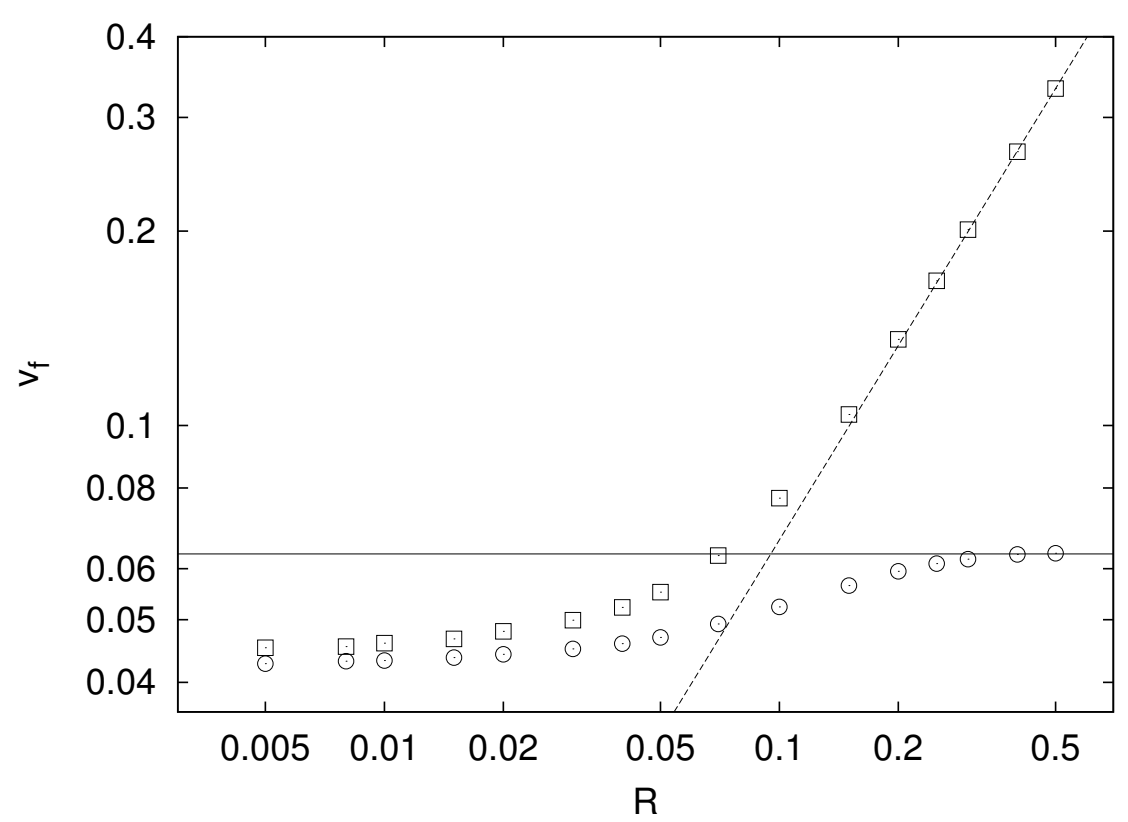

FIG. 9: ( $\square$ ) Front speed $v_{\mathrm{f}}$ versus $R$ and (o) the prediction of formula (17), and $p_{R}(N)$ computed in closed domains with initially separated particles distribution. The horizontal line is the value in the FKPP continuum limit, while the dashed line is the behaviour $v_{f} \simeq R$. The parameters are the same as Fig. 8. The Kuramoto length is $l_{k}=0.045$.

radius becomes larger than the Kuramoto length

$$
l_{K}=\sqrt{\frac{2 D}{p}},
$$

and therefore the continuum FKPP limit does not hold. Indeed, in particle systems, when $R \leq l_{k}$ the interaction term establishes a connection between regions containing $A$ particles and regions containing $B$ particles that in the classical FKPP equation could not be connected. Therefore, when $R \geq l_{K}$, it is not possible to obtain the continuum FKPP limit (1) even with an arbitrarily large number of particles. In Figure 10 it is shown the front speed at varying $R$ for various $N$. One can observe that at increasing $N$ for small $R$ the front speed approaches the FKPP value, while for large $R$ the front speed does not depend on $N$ and the value is definitely different from the FKPP value. 


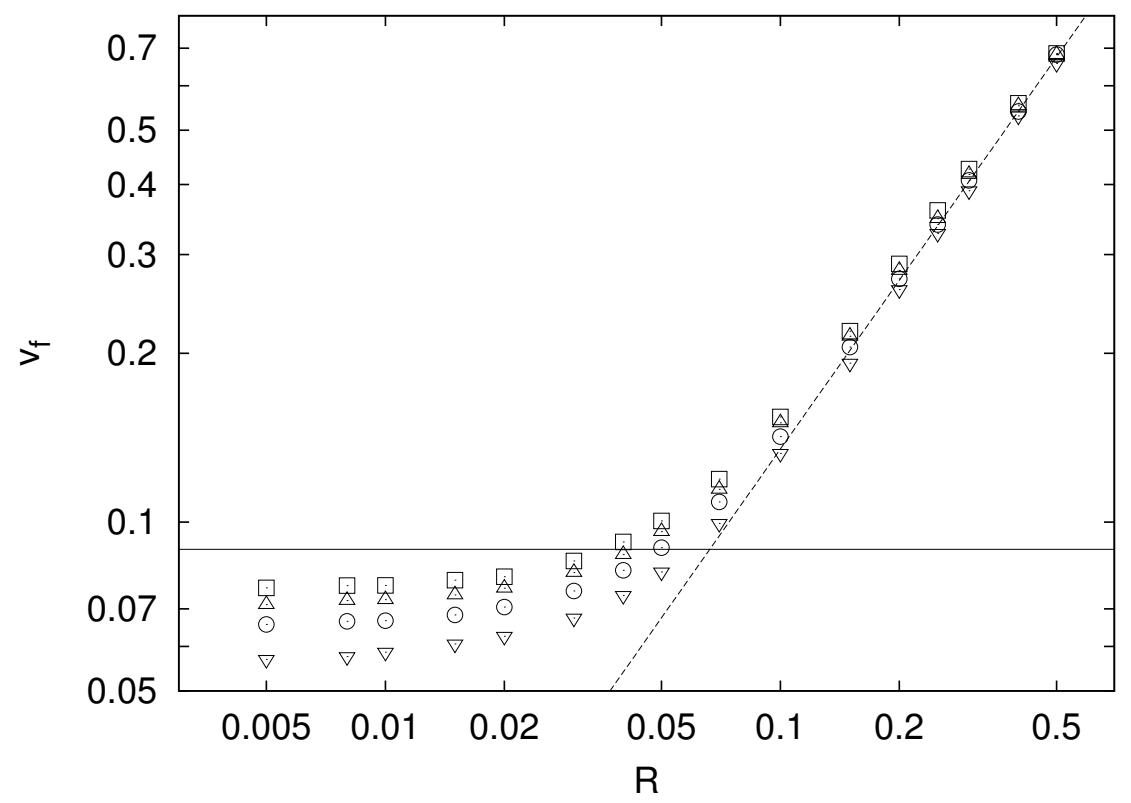

FIG. 10: The measured front speed $v_{\mathrm{f}}$ versus $R$ for $(\nabla) N=5000,(\circ) N=10000,(\square) N=20000$ and $(\triangle) N=40000$. The horizontal line is the value in the FKPP continuum limit, while the dashed line is the behaviour $v_{f} \simeq R$. The Kuramoto length is $l_{k}=0.045$.

A simple argument explains the behavior of $v_{\mathrm{f}}$ for large $R$. The front speed is proportional to the front length times the reaction rate, e.g., in the FKPP equation $v_{0}=2 \sqrt{D p} \propto$ $p \sqrt{D / p} \propto p l_{k}$. When the interaction radius is greater than the Kuramoto length it is reasonable to expect that the front length becomes proportional to $R$ and so the front speed:

$$
v_{\mathrm{f}} \propto p_{R}(N) R=\alpha R \quad \text { when } \quad R \gg l_{k},
$$

in agreement with the results shown in Figures 9, 10 and in Figure 11 for various $p$. In particular, in the inset of Figure 11 one can see the behaviour of $\alpha$ as a function of $p$ :

$$
\alpha(p)=a p
$$

where $a$ is a constant. This is not surprising because $p$ is the continuum limit for the reaction rate which is reached asymptotically by the particle system, i.e., $p_{R}(N) \rightarrow p$ for large $R$. 


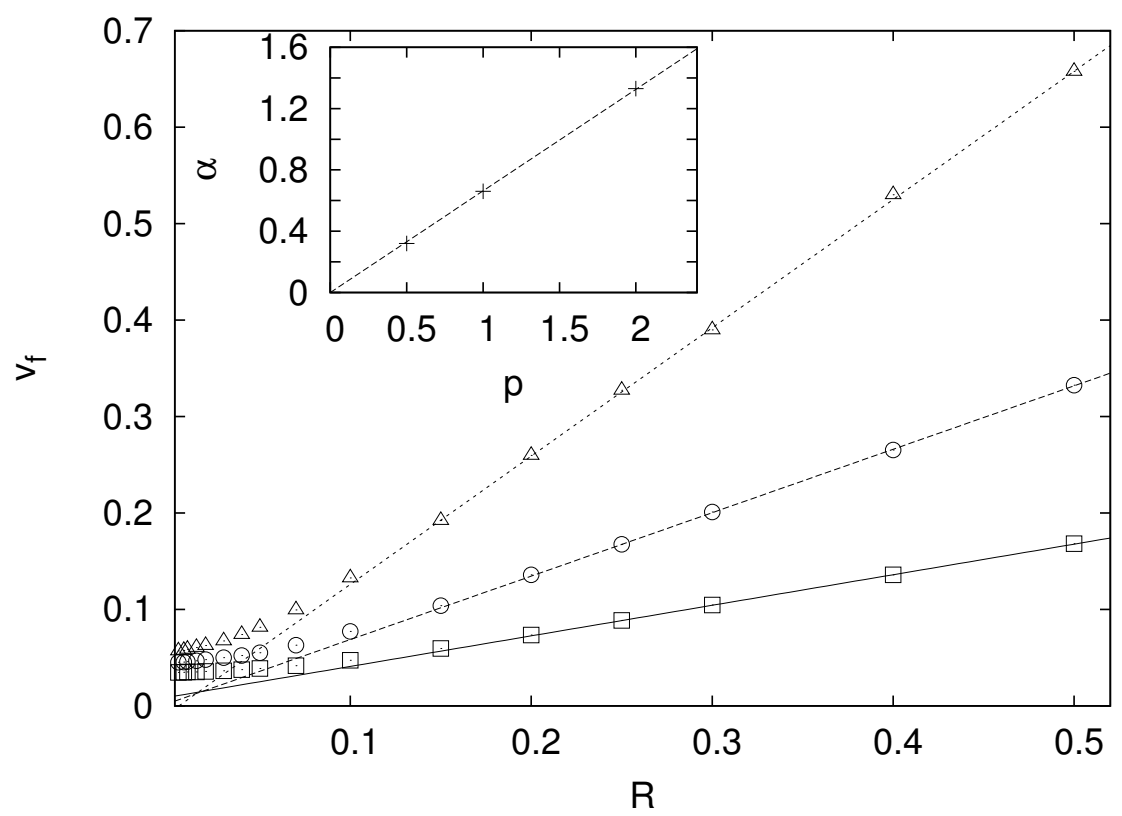

FIG. 11: Large $R$ behaviour of $v_{\mathrm{f}}$ in the cases: $p=0.5(\square), p=1.0(\circ)$ and $p=2.0(\triangle)$. In the inset the slope of the linear fit, $\alpha$ (see Eq. (20)), is shown as a function of $p$.

\section{B. Front thickness}

As a further confirmation of previous results, we investigate the behaviour of front thickness at varying $R$. Note that in the continuum limit there are many ways to compute the front thickness of a propagating front [20]. In the particle case, however, it is not obvious how to define a front profile. We proceed by defining an averaged field that resembles the front shape. Essentially this is a histogram over particle positions. Fixing our attention on $A$ particles, we define

$$
\tilde{\Theta}_{A}(x, \Delta x, t)=\frac{N_{x, \Delta x}(A, t)}{N \Delta x}
$$

where $N_{x, \Delta x}(A, t)$ counts the number of $A$ particles whose $x$ coordinate lays between $x$ and $x+\Delta x$. When the number of particles is large the value of $\Delta x$ could be taken arbitrarily small whereas, in general, $\Delta x$ has to be small, but at the same time large enough in order to avoid large fluctuations in $N_{x, \Delta x}(A, t)$. We use a relatively small $\Delta x$ (few $d_{m}$ ) and we average $N_{x, \Delta x}(A, t)$ over many different realizations. The front shape of an FKPP system 


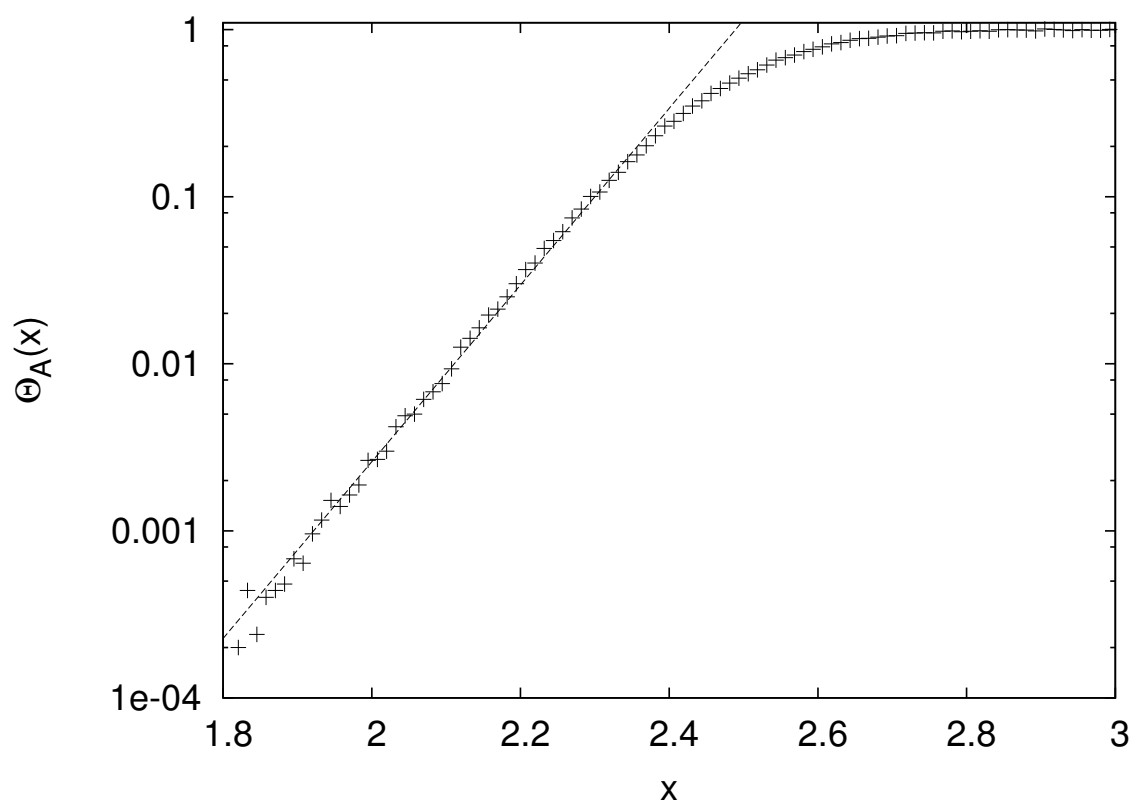

FIG. 12: The shape of the front $\tilde{\Theta}_{A}(x)(+)$ and the exponential fit of equation (22) (dashed line). behaves as

$$
\tilde{\Theta}_{A}(x, \Delta x ; t) \sim \exp \left(\left(x-x_{\mathrm{f}}(t)\right) / l_{A}\right),
$$

where $l_{A}$ is the front thickness, and $x_{\mathrm{f}}(t)$ the front position at time $t$. In Figure 12 it is shown the exponential behaviour of the front profile and the fit obtained from of Eq. (22). In particular Eq. (22) works well in the central region of the front, i.e., where corrections due to the particle nature of the system are less important.

Other measurements of the front profile provide similar results. In Figure 13 we plot the front thickness, $l_{A}$, computed for different values of $R$. Again, for $R$ smaller than the Kuramoto length the front thickness is constant, while for values of $R$ greater than $l_{k}$ the front thickness behaves as $l_{A} \propto R$. This result confirms the assumption of equation (19). The constant value reported in Figure 13 (the dashed line) is only an indicative value to show that for $R<l_{k}$ the front thickness is constant, and it is not the FKPP value of the front width. 


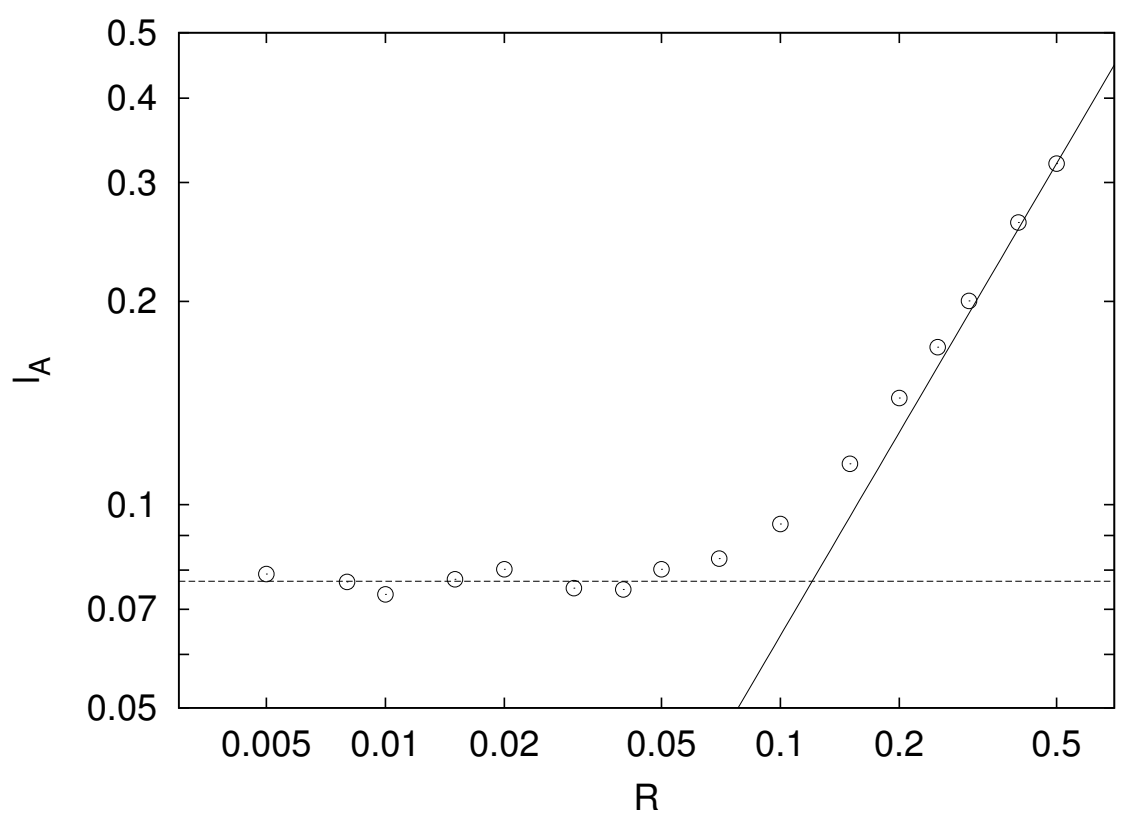

FIG. 13: Front thickness $l_{A}$ versus $R$ measured in the particle model (o). The dashed line corresponds to a constant value of the front speed (see fig.10), while the full line is the behaviour $l_{A} \simeq R$. The value of the Kuramoto length scale is $l_{K}=0.045$.

\section{SUMMARY AND CONCLUSIONS}

In this work we studied the effects of the discrete-particle character in an autocatalytic reacting system, described in terms of chemical dynamics where two types of Brownian particles interact when they are at a distance smaller than a certain radius $R$. We have shown that in a suitable continuum limit the system is equivalent to an FKPP model for the concentration of particles, and we have focused on the differences that arise when the conditions for this limit are not fullfilled.

The continuum limit holds if some relations among the relevant length scales (the interaction radius, the mean distance between particles and the Kuramoto length) of the problem are satisfied. For well-premixed initial conditions of the particles distribution only the first 
two lengths play a role. However, for front propagation, which requires a separated initial distribution of particles, also the Kuramoto length is important.

We have also considered the modified chemical dynamics $A+B \stackrel{p}{\longrightarrow} 2 B$, and $B \stackrel{q}{\longrightarrow} A$, where, at variance with the continuum limit, one can have the possibility of quenching, for small values of $R$. This is due to the particle nature of the model, which induces a qualitative difference with respect to the continuous description.

Moreover, in the context of front propagation, we have shown that particular conditions exist such that, increasing the particle density, the system reaches a continuum limit which is definitely different from the continuum FKPP limit.

We conclude noting that many biological systems are characterised by the two main ingredients of our work: the minimal distance for the interaction, and the exiguity of the number of organisms $[2,21]$. We hope that our work helps to clarify some shortcomings arising when a macroscopic description is attempted.

\section{ACKNOWLEDGMENTS}

We have benefited from a MEC-MIUR joint program (Italy-Spain Integral Actions). C.L. acknowledges support from FEDER and MEC (Spain), through project CONOCE2 (FIS2004-00953). A.V. and D.V. acknowledge support from PRIN-MIUR project "Dinamica Statistica di sistemi a molti e pochi gradi di libertà". We warmly acknowlege Emilio Hernández-García and Simone Pigolotti for a critical reading of the manuscript.

[1] J.D. Murray, Mathematical Biology, Springer-Verlag, Berlin (1993).

[2] G. Flierl, D. Grunbaum, S. Levin, and D. Olson, J. Theor. Biol. 196, 397 (1999).

[3] T. Tél, A. de Moura, C. Grebogi and G. Károlyi, Phys. Rep., 413, 91 (2005).

[4] A.N. Kolmogorov, I. Petrovskii and N. Piskunov, Bull. Univ. Moscow, Ser. Int. A, 1, 1 (1937).

[5] R.A. Fisher, Ann. Eugenics, 7, 353 (1937). 
[6] W. van Saarloos, Phys. Rep. 386, 29 (2003).

[7] J. Xin, SIAM Review 42, 161 (2000).

[8] D.G. Aronson and H.F. Weinberger, Adv. Math., 30, 33 (1978).

[9] W.R. Young, A.J. Roberts, G. Stuhne, Nature 412, 328 (2001).

[10] E. Hernández-García and C. López, Phys. Rev. E 70016216 (2004);

C. López and E. Hernández-García, Physica D 199, 223 (2004).

[11] C. R. Doering, C. Mueller, and P. Smereka, Physica A 325, 243 (2003).

[12] E. Brunet and B. Derrida, Phys. Rev. E 56, 2597 (1997).

[13] D. Panja, Phys. Rep. 393, 87 (2004).

[14] Y. Togashi and K. Kaneko, Phys. Rev. Lett. 86, 2459 (2001);

Y. Togashi and K. Kaneko, Phys. Rev. E 70, 020901 (2004).

[15] A.W. Visser and U.H. Thygesen, J. Plankton Res. 25, 1157 (2003); C. López, Phys. Rev. E 72, 061109 (2005).

[16] N. Vladimirova, P. Constantin, A. Kiselev, O. Ruchayskiy and L. Ryzhik, Combust. Theory Modelling 7, 487 (2003).

[17] J.M. Roquejoffre, Arch. Rat.Mech. Anal. 117, 119 (1992);

J.M. Roquejoffre, Ann. Inst. H. Poincaré, Anal. Nonlin. 14, 499 (1997).

[18] J. Mai, I.M. Sokolov, and A. Blumen, Europhys. Lett 44, 7 (1998);

J. Mai, I.M. Sokolov, and A. Blumen, Phys. Rev. E 62, 141 (2000).

[19] A. Lemarchand and B. Nowakovski, Europhys. Lett. 41, 445 (1998).

[20] M. Abel, A. Celani, D. Vergni and A. Vulpiani, Phys. Rev. E 64, 046307 (2001).

[21] R. Durrett and S.A. Levin, Theoretical Population Biology 46, 363 (1994). 